\title{
Phase-field method for epitaxial kinetics on surfaces
}

Joel Posthuma de Boer, lan J. Ford, Lev Kantorovich, and Dimitri D. Vvedensky

Citation: J. Chem. Phys. 149, 194107 (2018); doi: 10.1063/1.5049548

View online: https://doi.org/10.1063/1.5049548

View Table of Contents: http://aip.scitation.org/toc/jcp/149/19

Published by the American Institute of Physics

\section{Articles you may be interested in}

Unsupervised machine learning for detection of phase transitions in off-lattice systems. I. Foundations The Journal of Chemical Physics 149, 194109 (2018); 10.1063/1.5049849

Unsupervised machine learning for detection of phase transitions in off-lattice systems. II. Applications The Journal of Chemical Physics 149, 194110 (2018); 10.1063/1.5049850

Communication: Constrained molecular dynamics for polarizable models The Journal of Chemical Physics 149, 191102 (2018); 10.1063/1.5055704

A transferable artificial neural network model for atomic forces in nanoparticles

The Journal of Chemical Physics 149, 194101 (2018); 10.1063/1.5043247

On the calculation of the stress tensor in real-space Kohn-Sham density functional theory

The Journal of Chemical Physics 149, 194104 (2018); 10.1063/1.5057355

Global elucidation of broken symmetry solutions to the independent particle model through a Lie algebraic approach

The Journal of Chemical Physics 149, 194106 (2018); 10.1063/1.5049827

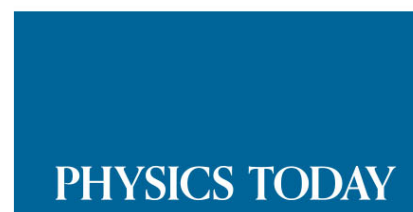

WHITEPAPERS
ADVANCED LIGHT CURE ADHESIVES

Take a closer look at what these environmentally friendly adhesive systems can do

\section{READ NOW}

PRESENTED BY

Q. MASTERBOND' 


\title{
Phase-field method for epitaxial kinetics on surfaces
}

\author{
Joel Posthuma de Boer, ${ }^{1}$ lan J. Ford ${ }^{2}$ Lev Kantorovich, ${ }^{3}$ and Dimitri D. Vvedensky ${ }^{1}$ \\ ${ }^{1}$ The Blackett Laboratory, Imperial College London, London SW7 2BZ, United Kingdom \\ ${ }^{2}$ Department of Physics and Astronomy and London Centre for Nanotechnology, \\ University College London, London WC1E 6BT, United Kingdom \\ ${ }^{3}$ Department of Physics, King's College London, The Strand, London WC2R 2LS, United Kingdom
}

(Received 23 July 2018; accepted 8 October 2018; published online 20 November 2018)

\begin{abstract}
We present a procedure for simulating epitaxial growth based on the phase-field method. We consider a basic model in which growth is initiated by a flux of atoms onto a heated surface. The deposited atoms diffuse in the presence of this flux and eventually collide to form islands which grow and decay by the attachment and detachment of migrating atoms at their edges. Our implementation of the phase-field method for this model includes uniform deposition, isotropic surface diffusion, and stochastic nucleation (in both space and time), which creates islands whose boundaries evolve as the surface atoms "condense" into and "evaporate" from the islands. Computations using this model in the submonolayer regime, prior to any appreciable coalescence of islands, agree with the results of kinetic Monte Carlo (KMC) simulations for the coverage-dependence of adatom and island densities and island-size distributions, for both reversible and irreversible growth. The scaling of the island density, as obtained from homogeneous rate equations, agrees with KMC simulations for irreversible growth and for reversible growth for varying deposition flux at constant temperature. For reversible growth with varying temperature but constant flux, agreement relies on an estimate of the formation energy of the critical cluster. Taken together, our results provide a comprehensive analysis of the phasefield method in the submonolayer regime of epitaxial growth, including the verification of the main scaling laws for adatoms and island densities and the scaling functions for island-size distributions, and point to the areas where the method can be extended and improved. Published by AIP Publishing. https://doi.org/10.1063/1.5049548
\end{abstract}

\section{INTRODUCTION}

Epitaxial phenomena have been studied for almost a century, ${ }^{1}$ not least because epitaxial growth is vital for the control of interfacial composition, structure, and integrity that underlies many important technologies based on heterogeneous interfaces. Examples include quantum-well and quantum-dot lasers, ${ }^{2}$ high-electron mobility transistors, ${ }^{3}$ and photodetectors. ${ }^{4}$ The exceptional transport properties ${ }^{5,6}$ and the concomitant widespread technological promise of graphene provided an impetus for the development of methods for producing large-scale quantities of this material. These efforts include experimental advances in graphene fabrication by numerous epitaxial techniques, as well as work by many groups striving to understand the atomistic mechanisms that govern graphene formation on various surfaces. ${ }^{7}$

Imaging and diffraction techniques have provided information over a range of length and time scales about the effects of particular epitaxial processes for various combinations of deposited materials and substrates. Such measurements have established a conceptual base for the development of theoretical and computational methods for understanding many aspects of morphological evolution..$^{8-11}$ Densityfunctional calculations provide ground-state energies and structures, ${ }^{12,13}$ and preferential absorption sites and configurations of adsorbed molecules, ${ }^{13}$ as well as information about kinetics, such as energy barriers for activated processes. ${ }^{14}$ Methods for the direct exploration of epitaxial kinetics range from molecular dynamics (MD) simulations, based on forces obtained from fully quantum mechanical calculations ${ }^{15}$ or empirical potentials, ${ }^{16}$ to coarse-grained stochastic methods. Among the latter, the most prevalent are kinetic Monte Carlo (KMC) simulations. ${ }^{17-20}$ In contrast to MD, which follow the trajectories of each constituent particle, KMC simulations treat the evolution of the system as a sequence of stochastic transitions between configurations specified by the occupancy of lattice sites in the system. Such simulations can be performed for much larger systems over far longer times than MD, which enables detailed comparisons to be made with experiments ${ }^{19}$ and scaling regimes studied at long times. $^{8}$

The main computation challenge for models of epitaxial growth is the incorporation of the broad range of length and time scales of processes that produce the desired surface morphology. ${ }^{21}$ Consider a typical experimental scenario during molecular-beam epitaxy. ${ }^{22}$ Atoms or simple homoatomic molecules are deposited onto a heated substrate. Deposition rates are typically of the order of 1 monolayer (ML) per second, representing the average arrival rate per site. Deposited atoms diffuse along the surface with an average residence time of $10^{-3}-10^{-6} \mathrm{~s}$ per site (the reciprocal of the hopping rate). Migrating adatoms collide to form dimers which 
grow/decay by the capture/emission of atoms, eventually forming a morphology whose structure depends on the choice of materials and growth conditions (deposition fluxes and temperature).

The growth of graphene on hexagonal substrates provides an extreme example of growth conditions that provide modeling challenges. Growth is initiated by several fast processes: adsorption and decomposition of precursor molecules, desorption of their fragments once carbon is released, and nucleation of clusters of carbon atoms, which grow by the attachment of migrating carbon atoms. ${ }^{23,24}$ Low deposition rates produce graphene growth times of macroscopic sheets that can extend over hours. Moreover, the high temperatures and rapid surface diffusion mean that simulating graphene growth can exceed the capabilities of even the most efficient algorithms. ${ }^{25,26} \mathrm{In}$ this case, reduced models, such as homogeneous rate equations, which describe the time-dependent spatially averaged concentrations of species on a substrate, ${ }^{27-29}$ provide practical alternatives.

One approach to addressing the challenges of modeling large-scale epitaxial growth is based on replacing the diffusion of discrete adatoms by a continuous field governed by the diffusion equation. ${ }^{30-32}$ A noteworthy implementation of this scheme is the "island dynamics" model. ${ }^{33}$ The BurtonCabrera-Frank (BCF) equations, ${ }^{34}$ which describe the growth and decay of epitaxial islands due to the attachment and detachment of adatoms, are solved by using the level-set method. ${ }^{35,36}$ A simple rate equation for the island density that depends only on the average adatom density determines nucleation times, with the position of a nucleated island chosen by a stochastic algorithm. Excellent agreement is obtained between experiment and KMC simulations for irreversible aggregation, where adatoms cannot detach from island edges, in the submonolayer regime. The method has been extended to account for adatom detachment from island edges (reversible aggregation), ${ }^{37}$ Ostwald ripening, ${ }^{38}$ and the effects of strain due to lattice mismatch. ${ }^{39}$

Here, we apply a somewhat different strategy by using the phase-field method to model the submonolayer growth of a monatomic crystal. The phase-field method is based on the Ginzburg-Landau theory of phase transitions and provides a mathematical description for moving boundary problems during phase transformations, such as solidification, in which the interface has a finite, but small, thickness. The central quantity in this method is an auxiliary function, called the "phase field," whose value identifies the phase at every point in space and time. The phase-field model of the solid-liquid phase transformation was first proposed by Langer ${ }^{40}$ and has become a widely used method for computing realistic morphologies in a variety of settings. ${ }^{41,42}$ For our purposes, this method offers several advantages over the level-set method, including the ability to treat irreversible and reversible aggregation within a single framework, that is, without resorting to ad hoc, albeit sophisticated, schemes. ${ }^{37}$

There have been several applications of the phasefield method to epitaxial systems, including growth in the submonolayer $^{4-45}$ and multilayer ${ }^{45,46}$ regimes, on stepped surfaces $^{47-49}$ and spirals, ${ }^{50}$ and on patterned surfaces. ${ }^{51}$ Submonolayer epitaxy, the focus of our interest here, has been the most extensively studied regime, both experimentally and theoretically, ${ }^{52}$ because scanning tunneling microscopy provides detailed information about island sizes and their distributions, which can be compared directly with predictions based on scaling theory to identify growth mechanisms and critical island sizes. Scaling theory yields power laws for adatom and island densities, ${ }^{53}$ scaling functions for distributions of island sizes, ${ }^{54-56}$ and the spatial correlations between island positions. ${ }^{57}$ Previous work has focused either on island-size distribution functions for reversible and irreversible aggregation, but with uncertain results for island density scaling, ${ }^{44}$ or solely on the scaling of island densities. ${ }^{43,45}$ Here, we carry out a detailed examination of phase-field calculations of submonolayer epitaxy by determining exponents for adatom and island densities and, crucially, island-size distributions and demonstrate the crossover of these quantities from irreversible to reversible aggregation.

The organization of this paper is as follows. In Sec. II, we show how the growth of islands can be described by the BCF equations. The phase-field equations, which provide solutions to the BCF equations, and their "thin interface limit" are developed in Sec. III. The nucleation of islands, which is an adjunct to the phase-field equations, is described in Sec. IV, including when and where dimers are placed on the surface and how they are incorporated into the phase field. The numerical implementation of our phase-field method is described in Sec. V. Results for irreversible and reversible aggregation are presented in Sec. VI. We have determined surface morphologies, island-size distributions, and the scaling of adatom and island densities. Agreement with KMC simulations is obtained for all of these quantities. For reversible growth with varying temperature but constant flux, agreement relies on an estimate of the formation energy of the critical cluster. Conclusions and an outline of directions for further development are provided in Sec. VII.

\section{THE MODEL}

We consider a model in which deposited adatoms are the only mobile surface species. All other species (dimers, trimers, etc.) are assumed to be immobile and are referred to as "islands" which can grow/decay by the gain/loss of adatoms at their edges. This model has been used to quantitatively account for several aspects of epitaxy on semiconduc$\operatorname{tor}^{58-60}$ and metal ${ }^{52}$ surfaces. However, for some materials, other species, such as clusters of atoms, can diffuse on the surface $^{29}$ and, hence, contribute to the nucleation and growth of islands. Mobile clusters and other extensions to our basic method will be discussed in Sec. VII.

The limit where no detachment of adatoms from islands occurs is known as irreversible growth or aggregation. ${ }^{61}$ This is the regime of low temperatures and/or high fluxes. This means that when two adatoms collide on the surface, they combine irreversibly to form an immobile dimer which then grows by capturing diffusing adatoms. Growth is "reversible" if adatoms can detach from island edges. In the language of classical nucleation theory, ${ }^{27}$ irreversible growth corresponds to a critical number $i=1$ of atoms, where islands with two atoms are stable and grow by capturing migrating atoms. 
Reversible growth corresponds to critical sizes $i \geq 2$, where islands with fewer than $i$ atoms tend to decay, while those with greater than $i$ atoms tend to grow. The assumption of irreversibility reduces the number of atomistic processes that must be considered. Although irreversible aggregation is somewhat idealized, there are materials ${ }^{10,52,62-64}$ that exhibit this growth mode.

The philosophy of coarse-grained models for epitaxial growth, such as the phase-field method used here, is to replace some of the discrete atomistic processes by continuous equations valid over large length and time scales. ${ }^{30-32}$ This is accomplished in part by introducing a continuous field $c(\mathbf{r}, t)$ for the mobile adatom density at position $\mathbf{r}$ on the surface at time $t$. Away from island boundaries, the evolution of $c(\mathbf{r}, t)$ is determined by the two-dimensional diffusion equation

$$
\frac{\partial c}{\partial t}=D \nabla^{2} c+J
$$

where $D$ is the adatom diffusion coefficient, assumed to be a constant across the surface, and $J$ is a continuous flux of adatoms onto the surface. In the following, we will work with the dimensionless quantity $n=\Omega c$, which is the number of atoms per atomic site with area $\Omega=a,{ }^{2}$ where $a$ is a lattice spacing. Multiplying (1) by $\Omega$ yields

$$
\frac{\partial n}{\partial t}=a^{2} \tilde{D} \nabla^{2} n+\tilde{J},
$$

in which $D=a^{2} \tilde{D}$, so $\tilde{D}$ is the hopping rate between the nearest neighbor sites separated by a distance $a$ and $\tilde{J}=\Omega J$ is the deposition rate per site. Both $\tilde{D}$ and $\tilde{J}$ have units of inverse time.

Island edges act as sources or sinks for the adatom density. The fluxes of adatoms at these edges determine the normal velocity $v_{n}=\mathbf{v} \cdot \hat{\mathbf{n}}$ of the island boundaries,

$$
v_{n}=a^{2} \tilde{D} \hat{\mathbf{n}} \cdot\left(\left.\nabla n\right|_{+}-\left.\nabla n\right|_{-}\right),
$$

for an interface with outward normal $\hat{\mathbf{n}}$. Here, the subscript + $(-)$ refers to the lower (upper) terrace at an island boundary (Fig. 1).

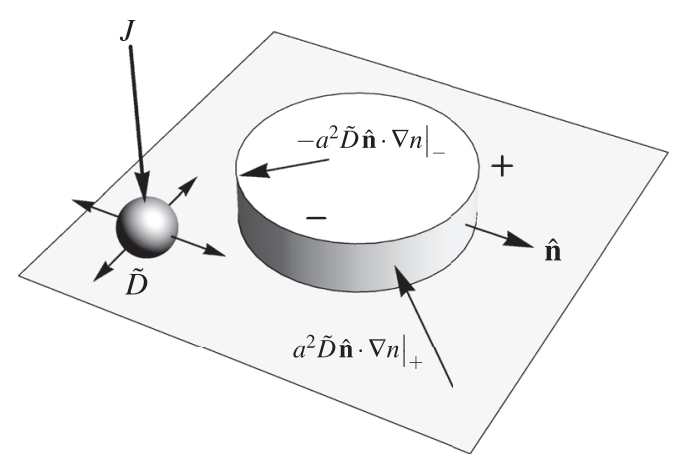

FIG. 1. Schematic diagram for a system described by the BCF equations with upper ("-") and lower ("+") terraces (the latter shown shaded) separated by the sharp interface of an island edge. Adatoms are deposited onto the surface (including the top of islands) at a rate of $\tilde{J}$ per lattice site, and then migrate with the hopping rate $\tilde{D}$ between the nearest neighbor lattice sites separated by $a$. Arrows to and from the interface show the flux from the upper and lower terraces to the interface with outward normal $\hat{\mathbf{n}}$ [Eq. (3)].
The curvature of the interface between the island and the mobile adatoms is accounted for by an approximate form of the Gibbs-Thomson equation ${ }^{65}$ appropriate for fast adatom detachment at step edges. This effectively assumes that the adatoms near a step edge are dilute enough to be regarded as mutually noninteracting. In this case, the number of adatoms outside an island of radius $R$,

$$
n_{\text {step }}=n_{\text {eq }}^{0} \exp \left(\frac{\gamma \Omega \kappa}{k_{B} T}\right) \approx n_{\mathrm{eq}}^{0}\left(1+d_{0} \kappa\right)
$$

is enhanced relative to the equilibrium adatom density $n_{\mathrm{eq}}^{0}$, where

$$
d_{0}=\frac{\gamma \Omega}{k_{B} T}
$$

is the capillary length, $\gamma$ is the step free energy per unit length (assumed to be isotropic and independent of curvature), $\kappa$ is the local curvature of the interface, $k_{B}$ is Boltzmann's constant, and $T$ is the absolute temperature of the substrate. Equation (4) serves as the boundary condition for (1) at the interface. Equations (1)-(4) are referred to as the BCF equations. ${ }^{34}$

The capillary length (5) determines the effect of curvature on the growth and decay of islands. For $d_{0}$ small compared to the radius of an island $\left(d_{0} \kappa \ll 1\right)$, capillarity has little influence, and islands grow at a rate proportional to the supersaturation of the adatom density. However, capillarity becomes important for larger $d_{0}$, and islands below a critical radius tend to decay unless the surrounding adatom density is high enough to enable detachment to be balanced or exceeded by attachment.

The role of $n_{\text {eq }}^{0}$ in (4) can be understood by examining reversibility in relation to the boundary conditions of the BCF equations solved by (12) and (14) in the thin interface limit (Sec. III B). These points are discussed in Ref. 37, but we repeat the main elements of the argument here. The key observation is that, if growth is irreversible, island boundaries act as perfect sinks and the diffusion equation is solved subject to the absorbing boundary condition

$$
\left.n(\mathbf{r}, t)\right|_{\text {step }}=0 .
$$

If adatoms can detach from island boundaries, this condition becomes

$$
\left.n(\mathbf{r}, t)\right|_{\text {step }}=n_{\text {step }}^{(0)},
$$

where $n_{\text {step }}^{(0)}$ is the equilibrium density for an island of radius $R$. For large circular islands,

$$
\kappa=\frac{1}{R}
$$

and $n_{\text {step }}^{(0)}$ is given by the corresponding Gibbs-Thomson relation (4), which is satisfied by the phase-field equations in the thin interface limit (Sec. III B). The Gibbs-Thomson relation implies that, on a surface with islands of various curvatures, a concentration gradient is established whereby adatoms diffuse from interfaces with large curvature to interfaces with small curvature, that is, from small islands to neighboring large islands. This coarsening process is known as Ostwald ripening $^{66}$ and is particularly effective during equilibration, when there is no particle source. 
This argument highlights two features of our model. First, the assumption that the linearized Gibbs-Thomson equation is valid for islands containing as few as two atoms, the smallest islands in our model. This approximation captures enough of the curvature effects to produce the crossover between irreversible and reversible growth, as will be shown below. Second, for irreversible aggregation, the phase-field equations do not reproduce the boundary condition (6) exactly in the thin interface limit. However, for $a n_{\mathrm{eq}}^{0} d_{0} \ll 10^{-6}$, agreement is obtained with previous models of irreversible aggregation. We conclude that errors resulting from the boundary condition in (6) are negligible.

\section{THE PHASE-FIELD METHOD}

\section{A. Solution of BCF equations}

The BCF equations constitute a "Stefan problem," in which moving boundaries separate two phases (in our case, mobile adatoms and their condensed phase, which are islands). The motion is specified by quantities on either side of the boundary, that is, within the two phases. The phase-field method enables us to solve the BCF equations without having to keep track of the motion of interfacial boundaries.

In phase-field formulations of epitaxial growth, an auxiliary field called the "phase field" and denoted by $\phi(\mathbf{r}, t)$ is introduced to describe the position and spatial extent of immobile islands on the surface of the substrate. The phase field varies continuously between regions where $\phi=1$, indicating the presence of islands, and regions where $\phi=0$, which is associated with the presence of the adatom field $n(\mathbf{r}, t)$.

The equation of motion for the phase field is derived by first postulating a (dimensionless) "free energy" functional $F[\phi(\mathbf{r}, t)]$ of the phase field. The choice of this functional depends on the problem at hand and is typically based on phenomenological considerations. A common choice is the sum of a "bulk free energy," which in a two-phase system is a double-well potential, a gradient term representing the free energy cost of spatial variations between the two phases, such as interfaces, and a term to account for interactions between the phase field and any other fields. If $\phi$ is not conserved locally, its time dependence can be obtained by assuming dissipative dynamics,

$$
\tau \frac{\partial \phi(\mathbf{r}, t)}{\partial t}=-\frac{\delta F}{\delta \phi}
$$

where $\tau$ is a time scale that determines the relaxation of the phase field to equilibrium and the right-hand side is the functional derivative of $F$ with respect to $\phi$.

The free energy used here ${ }^{43}$ is an extension of the expression proposed by Liu and Metiu ${ }^{67}$ and has been used in other studies of epitaxial growth ${ }^{44,45}$ on initially flat surfaces,

$$
\begin{aligned}
F[\phi(\mathbf{r}, t)]= & \int \frac{d^{2} \mathbf{r}}{\Omega}\left\{\frac{W^{2}}{2}(\nabla \phi)^{2}-\frac{1}{\pi} \cos (2 \pi \phi)\right. \\
& \left.+\lambda\left(n-n_{\mathrm{eq}}^{0}\right)\left[\frac{1}{\pi} \sin (2 \pi \phi)-2 \phi\right]\right\},
\end{aligned}
$$

where $W$ is the width of the interface between regions where $\phi=1$ and where $\phi=0, \lambda$ is a dimensionless coupling constant, and $u_{\mathrm{eq}}^{0}$ is the equilibrium adatom number density at a straight step,

$$
u_{\mathrm{eq}}^{0}=e^{-\beta E_{0}},
$$

in which $E_{0}$ is the energy difference between a free adatom and an atom at a step and $\beta=1 /\left(k_{B} T\right)$.

The second term on the right-hand side of (10) is the "bulk free energy," which has degenerate minima at $\phi=0,1,2, \ldots$. The first term is the free energy cost of creating an interface between regions with different values of $\phi$, which corresponds to an island boundary (Fig. 1). The third term couples the phase field and the adatom density through the supersaturation $n-n_{\mathrm{eq}}^{0}$ and causes the island boundary to advance or retract by the capture or release of adatoms, depending on the sign of the supersaturation.

The number of adatoms per site is determined by a diffusion equation similar to (1),

$$
\frac{\partial n}{\partial t}=a^{2} \tilde{D} \nabla^{2} u-\frac{\partial \phi}{\partial t}+\tilde{J}-2 \Omega \sum_{k} \delta\left(\mathbf{r}-\mathbf{r}_{k}\right) \delta\left(t-t_{k}\right) .
$$

The first term on the right-hand side accounts for adatom diffusion on the terraces between island edges. The diffusion coefficient has the Arrhenius form obtained from transition-state theory,

$$
\tilde{D}=v e^{-\beta E_{D}},
$$

where $v=2 k_{B} T / h \sim 10^{11}-10^{13} \mathrm{~s}^{-1}$ is an attempt frequency, $h$ is Planck's constant, and $E_{D}$ is the energy barrier for hopping between the nearest lattice sites. The second term in (12) accounts for the capture of adatoms by existing islands. The third term corresponds to the uniform deposition rate of atoms per lattice site $\tilde{J}$ of atoms onto the substrate. The fourth term accounts for the loss of two atoms with each nucleation event of a dimer. Studies ${ }^{68,69}$ of epitaxial processes in such models have revealed that, in the early stages of growth, island nucleation is the dominant source of noise, while deposition noise is negligible. Hence, we take deposition to be a uniform deterministic process.

The equation of motion for the phase field is obtained from (9) and (10),

$$
\tau \frac{\partial \phi}{\partial t}=W^{2} \nabla^{2} \phi-2 \sin (2 \pi \phi)-2 \lambda\left(n-n_{\mathrm{eq}}^{0}\right)[\cos (2 \pi \phi)-1] .
$$

The evolution of an island boundary, which we take as the contour $\phi=\frac{1}{2}$, is then determined by the solution of the coupled equations (12) and (14). This system of equations corresponds to the "isothermal variational formulation," 70 in which the two equations are not derived from a single free energy functional. Instead, the second term in the right-hand side of (12), which describes the gain/loss of adatoms by islands, is entirely phenomenological. The relation to the $\mathrm{BCF}$ equations is discussed in Sec. III B.

In previous implementations ${ }^{43-45}$ of the phase-field method for submonolayer epitaxy, deposition was based on 
the random arrival of adatoms, and the nucleation rate of new islands was derived from the estimate $\lambda_{n} n^{2}$ obtained from rate equations, ${ }^{27,28,52}$ where $\lambda_{n}$ is an inverse time scale. This term is added to (14) as a new coupling for the phase field. By contrast, we regard nucleation as a discrete stochastic event in both space and time. Details are explained in Sec. IV.

\section{B. Thin interface limit}

The connection between the parameters in the phase-field formulation and the BCF equations is provided by the thin interface analysis. ${ }^{70}$ We define solutions $\phi_{\text {in }}, n_{\text {in }}, \phi_{\text {out }}$, and $n_{\text {out }}$ to (12) and (14), which are valid in an "inner region" near the interface and an "outer region" far away from the interface. In the outer region, $\phi_{\text {out }}$ is a constant determined by the minimum of the free energy, and $n_{\text {out }}$ satisfies the diffusion equation with source $\tilde{J}$ because the source/sink term $-\partial \phi / \partial t$ in (12) then vanishes. Equations for $\phi_{\text {in }}$ and $n_{\text {in }}$ are obtained by transforming a local coordinate system into a reference frame that moves with the velocity of the interface. Both $\phi_{\text {in }}$ and $n_{\text {in }}$ are then expressed as asymptotic expansions of the form

$$
\phi_{\text {in }}=\phi_{0, \text { in }}+\varepsilon \phi_{1, \text { in }}+\varepsilon^{2} \phi_{2, \text { in }}+O\left(\varepsilon^{3}\right),
$$

where $\varepsilon=W / \ell_{c}$ is a small dimensionless parameter and $\ell_{c}$ is the diffusion length. These equations are solved order by order subject to matching conditions for the inner and outer solutions at the interface. Equation (3) is recovered at $O(\varepsilon)$. At $O\left(\varepsilon^{2}\right)$, an expression is obtained for $n(\mathbf{r}, t)$ at the interface which, when compared with (4), provides relationships between the parameters in the phase-field and BCF equations. In the limit of instantaneous adatom attachment to island edges,

$$
\tau=\frac{c_{1} c_{2} W^{3}}{n_{\mathrm{eq}}^{0} d_{0} a^{2} \tilde{D}}, \quad \lambda=\frac{c_{1} W}{n_{\mathrm{eq}}^{0} d_{0}},
$$

where $c_{1}$ and $c_{2}$ are the numerical constants that depend on the free energy functional. For $F$ in (10), $c_{1}=0.36$ and $c_{2}=0.51$. $^{43}$

\section{NUCLEATION}

There are two ways of stipulating when a nucleation event occurs. Nucleation can be considered to be deterministic in time and based upon solving a rate equation,

$$
\frac{d N}{d t}=\sigma_{1} \tilde{D}\langle n(\mathbf{r}, t)\rangle^{2}
$$

for the number $N$ of islands per lattice site during irreversible aggregation, as was done in Ref. 33. This equation assumes that an island is formed when two adatoms meet at the same point $\mathbf{r}$. Hence, we regard the formation of a dimer as synonymous with the formation of an island. Here, $\sigma_{1}$ is the adatom capture number that represents the collision cross section, ${ }^{27,28,71}$

$$
\sigma_{1}=\frac{4 \pi}{\ln \left(\pi\langle n\rangle \frac{\tilde{D}}{\tilde{J}}\right)}
$$

Equation (17) is integrated over time, with a nucleation event occurring whenever the quantity $N L^{2} / \Omega$ passes the next integer. Here, $L^{2}$ is the area of the square domain used in the simulations, so $L^{2} / \Omega$ is the corresponding number of lattice sites.

Alternatively, nucleation events can be regarded as stochastic in time. In this case, at each time step, we form the quantity

$$
\sigma_{1} \tilde{D}\langle n(\mathbf{r}, t)\rangle^{2} \frac{L^{2}}{\Omega}
$$

which is the mean rate of dimer production on the substrate. This is similar to the expression used in rate equations (without the factor $L^{2} / \Omega$ ) for the average production rate of dimers per site. Assuming that the nucleation events are Poisson-distributed, the mean waiting time between nucleation events is

$$
\Delta t=-\frac{\Omega \ln (Y)}{\sigma_{1} \tilde{D}\langle n(\mathbf{r}, t)\rangle^{2} L^{2}},
$$

where $Y$ is a uniform random deviate in the range $[0,1]$. A nucleation event occurs whenever $\Delta t<d t$, where $d t$ is the time step. In our simulations, we regard nucleation as stochastic in time. The introduction of temporal fluctuations into the nucleation rate is not expected to have a large effect on our results. $^{72}$

Methods for choosing the position of the nucleated dimer have been explored ${ }^{72}$ with the level-set method. The best agreement with island-size distributions obtained from KMC simulations of irreversible aggregation in the pre-coalescence regime was found when the position of the nucleated dimer was chosen randomly and weighted by the local value of $n^{2}(\mathbf{r}, t)$. This ensures that islands are more likely to nucleate in the regions of high adatom density.

Once the position $\mathbf{r}_{0}$ of the nucleated dimer has been selected, we must increase the value of $\phi$ around this point. We do this by adding to the phase field an isotropic distribution $\tilde{\phi}$ centered at $\mathbf{r}_{0}$, which represents a new dimer. We use

$$
\tilde{\phi}(r ; z)=\frac{1}{2}\left[1-\tanh \left(\frac{r-z}{W}\right)\right],
$$

where $r$ is the radial distance from $\mathbf{r}_{0}$ and $z$ is a length determined by the condition that two atoms correspond to $\tilde{\phi}$. Transforming to polar coordinates and carrying out the azimuthal integral, we obtain

$$
2 \pi \int_{0}^{\infty} r \tilde{\phi}(r ; z) d r=\frac{\pi W^{2}}{2} \int_{0}^{-\exp (2 z / W)} \frac{\ln (1-s)}{s} d s=2 a^{2}
$$

The second integral is a representation of (minus) the dilogarithm function $L_{2},{ }^{73}$ which yields a transcendental equation for $z$,

$$
L_{2}\left(-e^{2 z / W}\right)=-\frac{4 a^{2}}{\pi W^{2}} .
$$

For $W=a$, this equation yields $z=0.27 a$ [Fig. 2(a)]. In the limit $W \rightarrow 0, \tilde{\phi}$ corresponds to a circular island with radius $r=a \sqrt{2 / \pi}$ [Fig. 2(d)]. To ensure mass conservation, dimer nucleation is accompanied by the simultaneous depletion of 

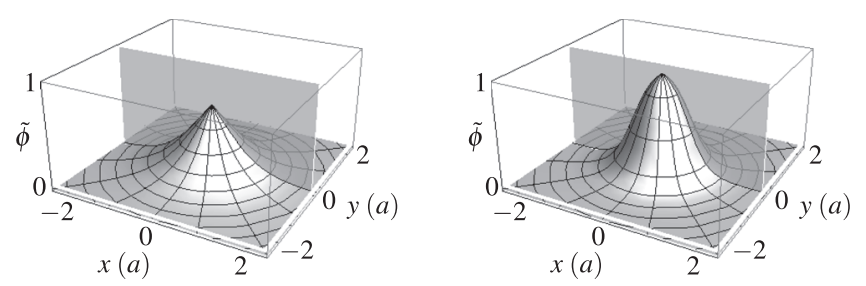

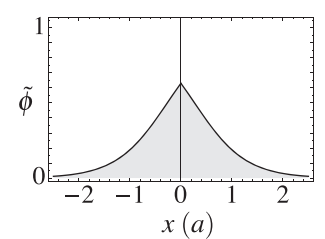

(a)
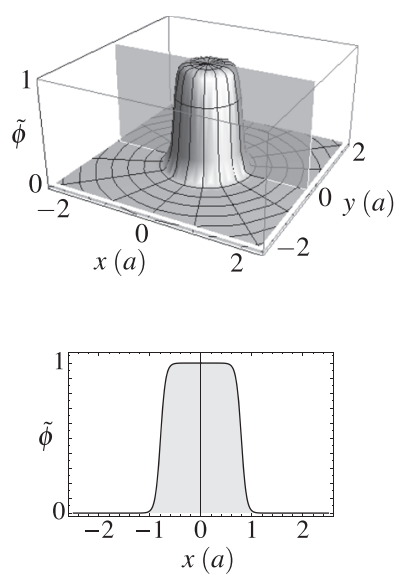

(c)

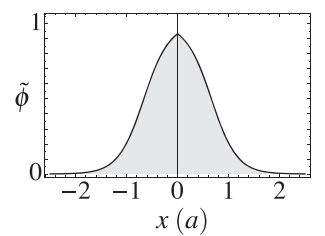

(b)
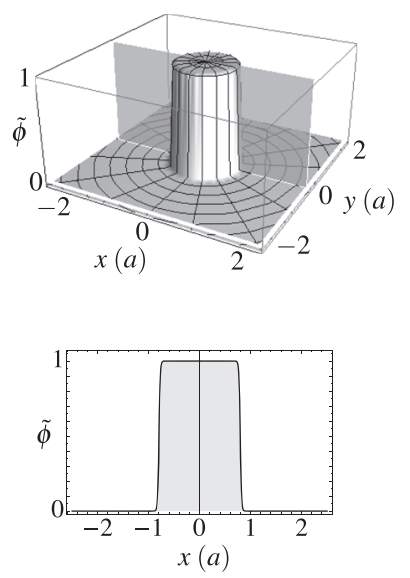

(d)
FIG. 2. Profiles of the phase field of nucleated dimers determined by (21) and (22) for interface widths of (a) $W=a$, (b) $W=0.5 a$, (c) $W=0.1 a$, and (d) $W=0.025 a$. Top panels show three-dimensional phase fields $\tilde{\phi}$, and bottom panels show the corresponding sections through the $x-\tilde{\phi}$ plane (shown shaded) in the top panels. Shaded regions in the bottom panels indicate the integral of the corresponding section of the profile. The spatial integral (22) of $\tilde{\phi}$ corresponds to two atoms.

the adatom density by an amount equal to the nucleated dimer. Similarly, dimer dissociation depletes the island density, with a concomitant increase of the adatom density field.

\section{NUMERICAL IMPLEMENTATION}

The phase-field equations are solved numerically on a grid. The main computational challenge is due to the large value of $D$ (typically, $10^{4}-10^{7} a^{2} s^{-1}$ ), which makes explicit finite difference methods prohibitively expensive. There are several ways of reducing the computing time. First, (12) and (14) can be solved with semi-implicit methods, which are usually a combination of implicit methods and operator splitting to account for non-linear terms. Second, finite differences can be retained, but the problem reformulated to facilitate parallelization, as in domain decomposition. ${ }^{44} \mathrm{We}$ use the former approach and implement exponential time differencing. ${ }^{74}$

Unless stated otherwise, the phase-field equations are solved on an $L_{N} \times L_{N}$ square grid with $L_{N}=512$ and spacing $\Delta x=\frac{1}{2} a$ such that the physical length of one side of the square domain is $L=\Delta x \times L_{N}=256 a$. Averages are obtained over 20-30 independent simulations. For most simulations, we set $\tilde{J} L / \Omega=1 \mathrm{ML} / \mathrm{s}$ and vary the temperature, but we also examine the effect on island densities of fixing the temperature and varying $\tilde{J}$. The interface width is set equal to $a$, but we have performed spot checks with smaller widths to verify that the thin interface limit has been reached.

We use the hopping barrier $E_{D}=1.4 \mathrm{eV}$ for all simulations, with other parameters indicated in the figure captions. A barrier of $1.4 \mathrm{eV}$ (with a prefactor of $v \sim 2 k_{B} T / h$ ) is appropriate for basic models of growth on surfaces such as $\mathrm{GaAs}(001),{ }^{59}$ while those for metal (001) surfaces fall in the range of $0.5 \mathrm{eV}^{75,76}$ But these differences are transformed away by the scaling analysis based on rate equations $\mathrm{s}^{27,54-56}$ which, for irreversible growth, subsumes growth statistics into expressions parameterized by the ratio $\tilde{D} / \tilde{J}$, the coverage, and the critical island size.

For reversible growth, there is an additional parameter that measures ${ }^{56,77,78}$ the extent of adatom detachment from island edges. In KMC simulations of submonolayer epitaxial growth, reversibility is determined by the lateral energy barrier between adatoms. ${ }^{78}$ Such a parameter does not appear in the phase-field formulation. However, by varying $d_{0}$ and $n_{\text {eq }}^{0}$, we obtain results that account for the crossover between irreversible and reversible growth. During irreversible growth, adatoms cannot detach from islands, so we will use this terminology if an island cannot become smaller for a set of parameters. During reversible growth, however, islands can decay or evaporate entirely.

\section{RESULTS}

The phase-field calculations presented in this section have been obtained with the adatom hopping barrier $E_{D}$, the equilibrium adatom density $n_{\text {eq }}^{0}$ (through $E_{0}$ ), and the capillary length $d_{0}$ (through $\gamma$ ) chosen to show the transition between irreversible and reversible aggregation. The hopping barrier sets the temperature range by determining the hopping rate of adatoms, while $n_{\mathrm{eq}}^{0}$ and $d_{0}$ determine the adatom detachment rates from island edges. Thus, at low temperatures, adatom hopping is suppressed, which increases the adatom density on the terrace, but detachment is also suppressed, leading to many small stable islands, i.e., to irreversible growth. At high temperatures, the increased adatom hopping diminishes the adatom density on the terrace, which reduces the island nucleation rate, but increases the adatom detachment rate, which destabilizes small islands. This is the regime of reversible aggregation.

\section{A. Surface morphologies}

Irreversible aggregation is simulated with parameters $n_{\mathrm{eq}}^{0} d_{0} \sim 10^{-6} a-10^{-8} a$ such that $\tau \sim 1 s$ [cf. (16)]. This choice is motivated by simulations ${ }^{79,80}$ which demonstrate convergence to the thin interface limit. By keeping $n_{\mathrm{eq}}^{0}$ small, and choosing $d_{0}$ such that $n_{\mathrm{eq}}^{0} d_{0}<10^{-6} a$, islands are unable to diminish in size, and we find results consistent with irreversible growth. Larger values of $n_{\mathrm{eq}}^{0} d_{0}$ introduce the possibility that 
nucleated dimers are unstable and may decay, depending on the adatom density at the interface.

The key parameter for irreversible epitaxial growth is the dimensionless ratio $R \equiv \tilde{D} / \tilde{J}$ of the hopping rate to the deposition rate per lattice site area. The asymptotic analysis of rate equations $\mathrm{s}^{27,28,56,57}$ shows that, as $R \rightarrow \infty$, adatom and island densities scale with coverage and $R$ and island size distributions approach a universal scaling form. This will be developed further in Secs. VI B-VI D.

For the analysis of reversible aggregation, $R$ must be supplemented by a quantity that reflects the degree of reversibility. Although there are several choices, ${ }^{56,77,78,81}$ an analysis based on rate equations shows that the critical island size ${ }^{27}$ emerges as a simple and intuitive quantity. However, as discussed in Sec. VI C, the critical island size is a statistical quantity that reflects the stochastic nature of the growth process and is, therefore, rarely an integer.

Figure 3 shows snapshots of surface morphologies at $\theta=0.1 \mathrm{ML}$ for irreversible and reversible growth. Such morphologies provide the most detailed information about a simulation and are the basis for further analysis in Secs. VI B-VID. Most apparent for irreversible growth [Fig. 3(a)] is the large number of small islands and the close proximity of some of these islands to each other and to much larger islands. There is no mechanism for the dissolution of islands, so only coalescence at larger coverages can eliminate small islands.

The corresponding morphology for reversible growth [Fig. 3(b)] is qualitatively different from the irreversible case. There are substantially fewer, but larger, islands and their positions are less irregular. This is a well-known feature of reversible growth and results from nucleation in unfavorable positions being "corrected" by the dissolution of smaller islands and the subsequent incorporation of their constituent adatoms into neighboring islands.

As is particularly evident in Fig. 3(b), the islands are square. This is an effect of the grid and, in particular, our choice of the grid spacing $\Delta x=\frac{1}{2} a$. We do not expect any adverse effect on our results, as square islands have been found ${ }^{28,37}$ to produce the same submonolayer statistics as other compact shapes. This is borne out in the results presented in Subsections VI B-VI D.

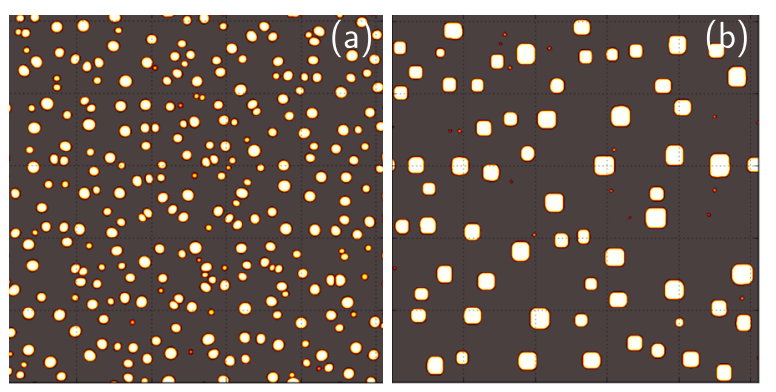

FIG. 3. Surface morphologies obtained from phase-field simulations of (a) irreversible aggregation $\left(E_{0}=1.2 \mathrm{eV}\right.$ and $\left.\gamma=10^{3} \mathrm{eV} / a\right)$ and (b) reversible aggregation $\left(E_{0}=0.7 \mathrm{eV}\right.$ and $\left.\gamma=0.5 \mathrm{eV} / a\right)$. For both simulations, $\tilde{D} / \tilde{J} \sim 10^{5}$, $L_{N}=512$, and $\theta=0.1 \mathrm{ML}$. The square shape of the islands is the result of the square grid with spacing $\Delta x=\frac{1}{2} a$ used for the numerical solution of the phase-field equations.

\section{B. Adatom and island coverages}

The coverage dependence (equivalent to time-dependence through $\theta=\widetilde{J} t L^{2} / \Omega$ ) of adatom and island coverages is shown in Fig. 4. For irreversible aggregation, the adatom coverage increases sharply after the initiation of the deposition flux, followed by a rapid decrease at the onset of island nucleation. Island coverage shows a continuously decreasing slope, with the steady state attained near 0.1 ML (not shown). The extended nucleation regime is evident in Fig. 3(a), with the morphology showing many small islands, some in close proximity to one another. There are also regions with few islands, which are favorable for dimer formation, as noted above.

The coverages of adatoms and islands during reversible aggregation is qualitatively different from the irreversible case, as Fig. 3 suggests. The initial peak in the adatom profile is shifted to slightly higher coverages because island formation is a slower process, due to the greater adatom coverage needed to form stable islands. Indeed, the coverage profile of the islands shows a maximum near 0.015 ML [Fig. 3(b)], followed by a saturation regime where there is little additional nucleation.

Nucleation is a more difficult process during reversible growth, which is reflected in the much higher adatom and lower island density compared to irreversible aggregation. This also affects the spatial distribution of islands and their sizes. As Fig. 3(b) shows, the islands are generally farther apart, larger, and more regularly spaced than those in Fig. 3(a). However, there are also some small islands; this is an artifact of our algorithm and will be discussed in Sec. VI C.

\section{Island-size distributions}

An important measure of the ability of our method to accurately describe the spatial arrangement of epitaxial islands is the distribution of island sizes. Islands grow by accreting mass from the ambient adatom population. The availability of adatoms is determined by the local environment of an island, that is, the number and sizes of neighboring islands. Hence, any inaccuracy in the spatial arrangement of islands is reflected in
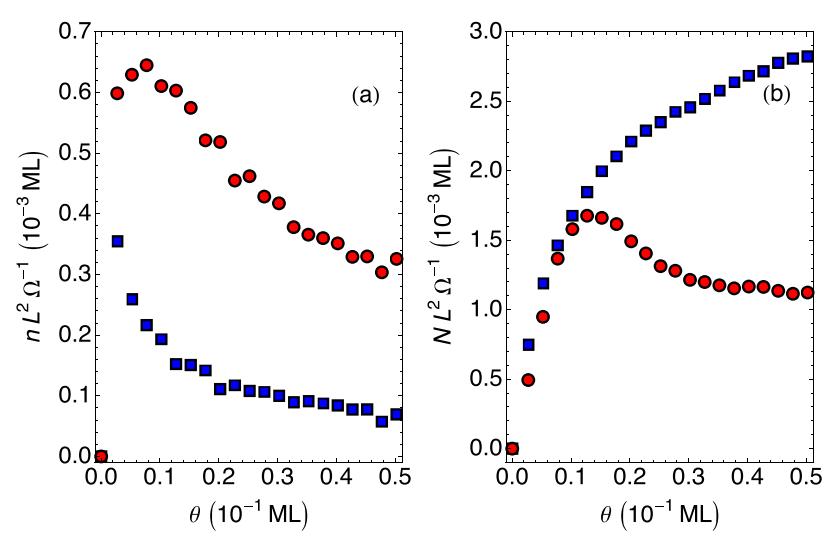

FIG. 4. Coverages of (a) adatoms and (b) islands for $0 \leq \theta \leq 0.05 \mathrm{ML}$ for irreversible (filled blue squares) and reversible (filled red circles) aggregation, with $\tilde{J} N / \Omega=1 \mathrm{ML} / \mathrm{s}$, so $\theta=t \mathrm{ML}$. For reversible aggregation, $E_{0}=1.5 \mathrm{eV}$ and $\gamma=10^{4} \mathrm{eV} / a$ while for irreversible aggregation, $E_{0}=2.5 \mathrm{eV}$ and $\gamma=10^{5} \mathrm{eV} / a$. In both cases, $\tilde{D} / \tilde{J}=6 \times 10^{5}$. 
the island-size distribution. The standard for such comparisons is based on KMC simulations, which capture all of the spatial correlations between islands and have been shown to quantitatively account for experimental measurements of island-size distributions. $^{62,63,81-85}$

To enable consistent comparisons across systems (simulated and real), the island-size data are plotted by using dimensionless scaled variables, which produces a data collapse onto a scaling function when the same mechanisms are operative. In particular, the number $N_{s}$ of $s$-atom islands per site prior to any appreciable coalescence of islands is $52,54-56$

$$
N_{s}=\frac{N}{s_{\mathrm{av}}} f\left(\frac{s}{s_{\mathrm{av}}}\right),
$$

where $N$ is the total number of islands per site, $f$ is a scaling function, and $s_{\mathrm{av}}$ is the average island size. A plot of $N_{s} s_{\text {av }} / N$ against $s / s_{\text {av }}$ yields the scaling function, which has normalization

$$
\int_{0}^{\infty} f(x) d x=1
$$

and first moment

$$
\int_{0}^{\infty} x f(x) d x=1 .
$$

Figure 5 shows island-size distributions obtained from our phase-field simulations for different values of $n_{\mathrm{eq}}^{0} d_{0}$ corresponding to reversible and irreversible growth. The symbols represent results of simulations with different temperatures at 0.1 ML, prior to any significant coalescence. The red lines are optimized analytic fits to KMC simulations for different critical island sizes. ${ }^{83}$ There is no change in the phase-field simulation used to obtain the distributions in Fig. 5, as they are determined solely by the values of $\gamma$ and $E_{0}$. This is in contrast to KMC simulations, where the increased number of diffusional hops per unit time leads to substantially greater computational overhead, while in level-set simula-
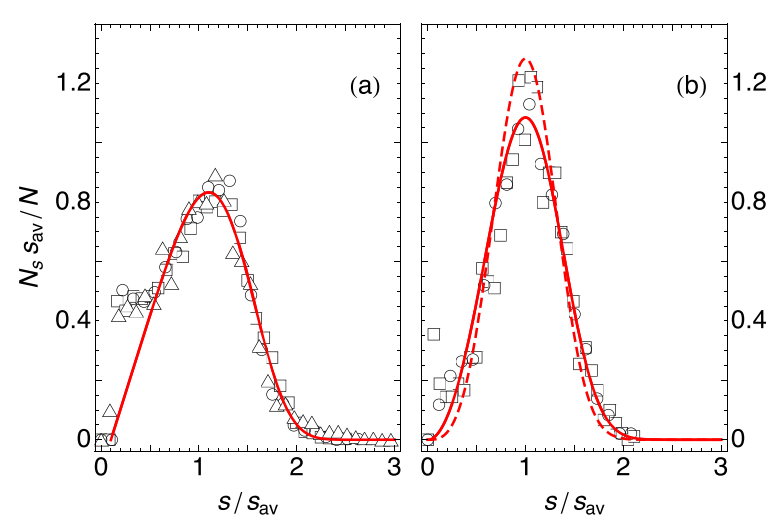

FIG. 5. Island-size distributions for (a) irreversible and (b) reversible aggregation at $950 \mathrm{~K}$ (circles) and $1000 \mathrm{~K}$ (triangles) plotted with scaled variables, as indicated in (24). Squares represent irreversible aggregation for $n_{\mathrm{eq}}^{0} d_{0} \sim 10^{-6}-10^{-8} a$ such that $\tau \sim 1 \mathrm{~s}$ (see text). Each symbol represents an average over 20-30 independent simulations at a coverage of $0.1 \mathrm{ML}$. The parameters for irreversible aggregation are $E_{0}=1.2 \mathrm{eV}$ and $\gamma=10^{3} \mathrm{eV} / a$ and for reversible aggregation are $E_{0}=0.7 \mathrm{eV}$ and $\gamma=0.5 \mathrm{eV} / a$. Red curves are optimized fits ${ }^{83}$ to KMC simulations for (a) $i=1$ and (b) $i=2$ (solid curve) and $i=3$ (broken curve). tions, a somewhat ad hoc procedure was adopted ${ }^{37}$ to account (successfully) for reversibility. Lowering the temperature in Fig. 5(a) takes the system deeper into the regime of irreversible aggregation, while raising the temperature in Fig. 5(b) takes the system toward larger values of the critical island size.

We consider the irreversible case first. Although the distribution follows the curve for a critical island size $i=1$ for $s / s_{\mathrm{av}} \gtrsim$ 0.5 , there are systematic deviations for smaller islands, which are manifested as a shoulder. Similar observations have been made for level-set calculations ${ }^{72}$ of irreversible aggregation. In fact, the same behavior is seen in rate equations and $\mathrm{KMC}$ simulations of "point" islands, that is, islands with no spatial extent, but which can still accumulate migrating adatoms in proportion to their size. ${ }^{55}$

The shoulder in Fig. 5(a) is due to the breakdown of our mean-field-like nucleation rule for the positions of new islands. An examination of Fig. 3(a) indeed reveals many small closely spaced islands and small islands near much larger islands. The growth rate of the small islands is diminished by the close proximity of neighboring islands, resulting in an over-estimate of the numbers of small islands.

The main effect of reversibility is an appreciable narrowing of the distribution. The solid and broken curves in Fig. 5(b) are obtained from optimized fits to KMC simulations ${ }^{83}$ with $i=2$ and $i=3$, respectively. Our parameters and growth conditions produce a scaling function between $i=2$ and $i=3$. This is typical of stochastic models of growth without a mandated critical cluster size, in which $i$ becomes a statistical quantity that reflects contributions from several critical island sizes. ${ }^{56,77,81}$ Only by requiring islands to have a specific critical size does the resulting scaling function correspond to that integer value. ${ }^{83}$

Another effect of reversibility is the much diminished shoulder for small island sizes. This effect, and the narrowing of the distribution, results from the system "correcting" inefficient nucleation sites by eliminating them by accretion to a neighboring larger island. Thus, there are fewer islands than in the irreversible case for the same coverage (Fig. 4), with a more uniform distribution of size and more regular spatial arrangement. The surface morphology in Fig. 3(b) confirms these observations.

Figure 6 compares the scaled island-size distributions in Fig. 5 with scaled experimental data obtained from homoepitaxial growth on $\mathrm{Fe}(001)$ and on GaAs surfaces. The lowtemperature $\left(20{ }^{\circ} \mathrm{C}\right)$ growth of $\mathrm{Fe}(001)$ and the growth of $\mathrm{GaAs}(110)$ at $480{ }^{\circ} \mathrm{C}$ both fall onto the distribution for irreversible aggregation. At higher temperatures $\left(356{ }^{\circ} \mathrm{C}\right)$, the growth of $\mathrm{Fe}(001)$ clearly indicates reversibility, as do the data for $\operatorname{GaAs}(111) \mathrm{A}$ at $480^{\circ}$. The data for Fe lie closer to the curve corresponding to $i=3$, as closer analysis of these data indicates, ${ }^{62}$ but the data for $\operatorname{GaAs}(111) \mathrm{A}$ indicate $i=2$, likely a result of the three-fold symmetry of this Ga-terminated surface. ${ }^{83}$ The important point about this figure is that, despite the differences in the structure, bonding, and growth kinetics of Fe and GaAs surfaces, scaling plots reduce the growth process to the most basic considerations, in these cases to the critical island sizes. This is the essence of the universality behind scaling laws. 

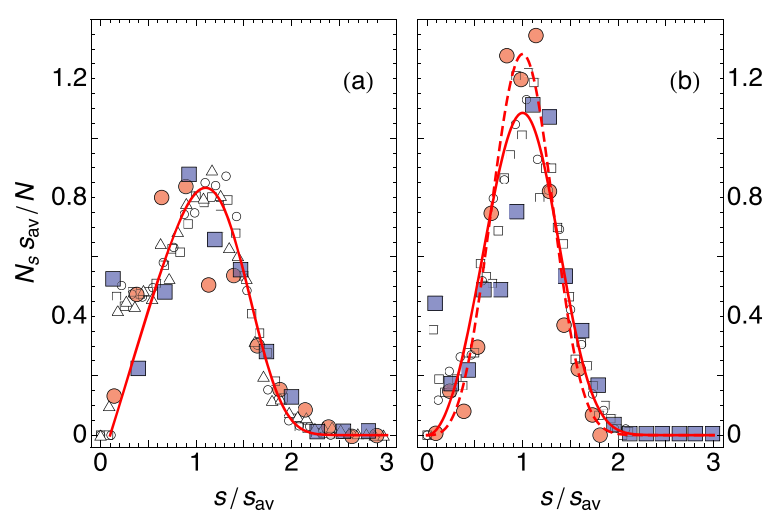

FIG. 6. Comparison of the scaled island-size distributions in Fig. 5 with experimental data of (a) homoepitaxy on $\mathrm{Fe}(001)$ at $20{ }^{\circ} \mathrm{C}$ (Ref. 62) (red circles) and homoepitaxial growth of $\mathrm{GaAs}(110)$ at $480{ }^{\circ} \mathrm{C}$ (Ref. 63) (blue squares) and (b) homoepitaxy on $\mathrm{Fe}(001)$ at $356^{\circ} \mathrm{C}$ (Ref. 62) (red circles) and homoepitaxial growth of $\mathrm{GaAs}(111) \mathrm{A}$ at $480^{\circ} \mathrm{C}$ [Ref. 63 (blue squares)].

\section{Scaling of adatom and island densities}

During the deposition of a new material, the numbers $n$ of adatoms and $N$ of islands per lattice site reach a quasi-steadystate regime in which rate equations predict scaling of adatoms and islands of the form ${ }^{52,53}$

$$
\begin{aligned}
& n \sim \theta^{-y_{i}} R^{\chi^{i}-1} \exp \left[\frac{E_{i}}{(i+2) k_{B} T}\right], \\
& N \sim \theta^{y_{i}} R^{-\chi_{i}} \exp \left[-\frac{E_{i}}{(i+2) k_{B} T}\right],
\end{aligned}
$$

for a critical island of size $i$, where $E_{i}<0$ is the binding energy of a critical island, $R=\tilde{D} / \tilde{J}$, and

$$
y_{i}=\frac{1}{i+2}, \quad \chi_{i}=\frac{i}{i+2} .
$$

These relations are derived by assuming that the mean capture number of stable islands is independent of coverage. In fact, capture numbers increase with coverage, ${ }^{86}$ resulting in the saturation of island densities (Fig. 4), that is, $y_{i}=0$. The scaling with $R$ has been verified by KMC simulations, ${ }^{52,78,87,88}$ but the scaling with coverage has been seen only for point islands.

Figures 7(a) and 7(b) show log-log plots of the adatom densities in Fig. 4(a) with best fits from a linear regression to the form

$$
\ln n=-y \ln \theta+\text { constant. }
$$

The conversion from the numbers of adatoms $n$ and islands $N$ per site into monolayers involves multiplication by the factor $L^{2} / \Omega$, which is the number of lattice sites (of area $\Omega$ ) in a system of size $L \times L$. For irreversible aggregation, we find $y$ $=0.79 \pm 0.01$, and for the reversible case, $y=0.52 \pm 0.02$. Both adatom densities are in the pre-saturation regime, so we do not expect to obtain $y=0$. However, our results are in agreement with those obtained from KMC simulations, ${ }^{78}$ which show increasing slopes with increasing lateral barriers. More detailed comparisons based on adatom densities would require additional calculations because of the different growth conditions and different parameterizations of attachment and detachment.

A $\log -\log$ plot of island densities as a function of $R$ is shown in Fig. 7(c) for irreversible aggregation, together with
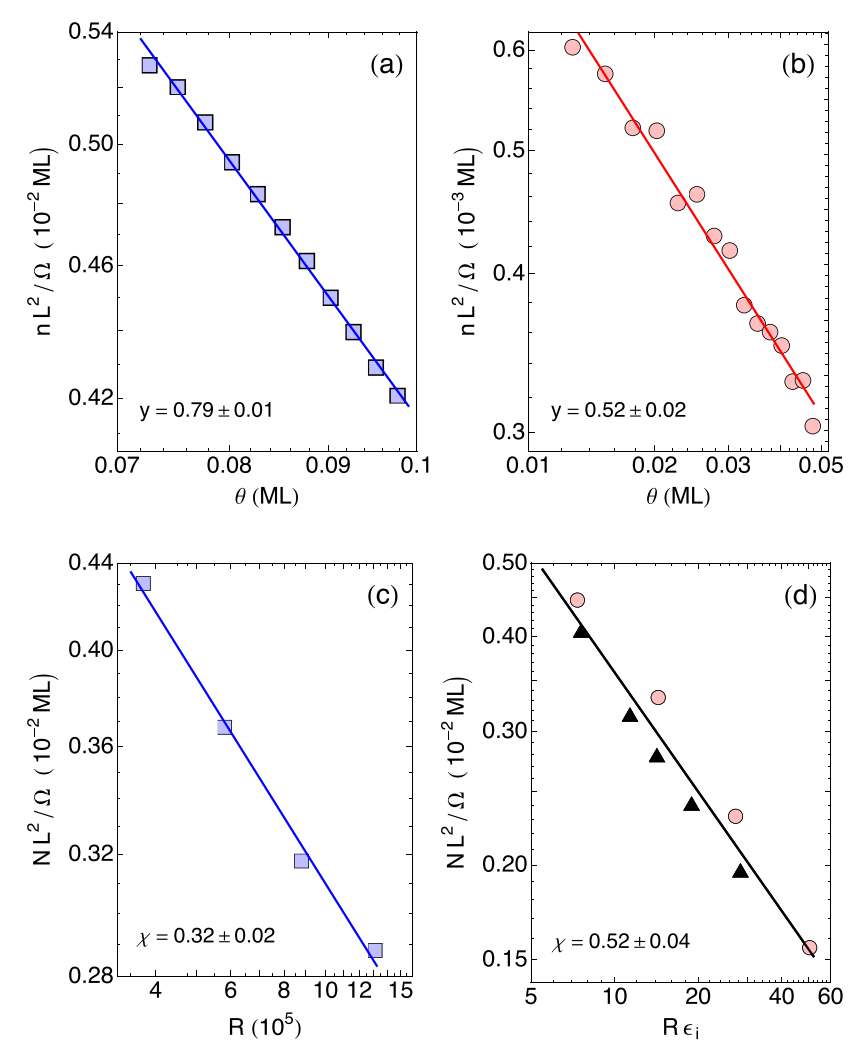

FIG. 7. Adatom density against coverage for (a) irreversible and (b) reversible aggregation for the data in Fig. 4(a). Straight lines are fits from a linear regression with slopes of (a) $y=0.79 \pm 0.01$ and (b) $y=0.52 \pm 0.02$. (c) Island density against $R$ for irreversible aggregation. (d) Island density against $R \epsilon_{i}$, where $\epsilon_{i}$ is given in (32), for variations in temperature at constant flux (filled red circles) and for variations in flux at constant temperature (filled black triangles). Straight lines are fits from linear regression with slopes of (c) $\chi=0.32 \pm 0.02$ and (d) $\chi=0.52 \pm 0.04$. Parameters for reversible aggregation are $E_{0}=0.8 \mathrm{eV}$ and $\gamma=0.3 \mathrm{eV}^{-1}$ and for irreversible aggregation are $E_{0}=2.6 \mathrm{eV}$ and $\gamma=10^{6} \mathrm{eV} a^{-1}$. Error bars are no larger than the symbol size.

the best fit from a linear regression. The value $\chi=0.32 \pm 0.02$ is in excellent agreement with $\chi_{1}=\frac{1}{3}$. This is not an altogether surprising result, as all KMC simulations obtain this slope for irreversible aggregation. However, while some phasefield implementations ${ }^{43}$ agree with this result, others ${ }^{44,45}$ do not.

We now consider the corresponding comparisons for reversible growth [Fig. 7(d)]. For this analysis, we write (28) as

$$
\ln N \sim \frac{i}{i+2} \ln \left(R \epsilon_{i}\right),
$$

where we have defined

$$
\epsilon_{i} \equiv \exp \left(\frac{\beta E_{i}}{i}\right)
$$

and we have omitted the coverage dependence since all data are taken at the same coverage. The factor $E_{i} / i$ in the exponential is the binding energy per atom in a critical island.

We have determined the island density by varying the growth conditions in two ways. We first varied the incident flux at constant temperature. In this case, the factor $\epsilon_{i}$ in (31) reduced to a constant and does not affect the scaling of the island density. We then varied the temperature at constant flux. Here, the factor $\epsilon_{i}$ does affect the scaling, which means that the ratio $E_{i} / i$ must be determined. Therefore, the quantity 
$E_{i} / i$ is varied until the slope of the second dataset (variable $T$ at constant $J$ ) is equal to that of the first (variable $J$ at constant $T$ ).

The results of our analysis are shown in Fig. 7(d). Linear regression applied to both sets of data, with a suitably chosen value of $E_{i} / i$ for the first set of data, yields the slope $\chi=0.52$ \pm 0.04 , which lies between $\chi_{2}$ and $\chi_{3}$. Indeed, by using the second equation in (29), we obtain $i=2.2 \pm 0.35$, which is consistent with Fig. 5(b) in that the island-size distribution lies between the curves for $i=2$ and $i=3$. Taking this analysis further would require calibrating a phase-field against KMC simulations to obtain effective values of binding energies for critical islands based on detachment barriers that reflect local environments.

\section{SUMMARY AND FUTURE WORK}

We have described the simulation of submonolayer epitaxial growth by using the phase-field method to solve the BCF equations in the presence of island nucleation. Where direct comparisons can be made, our results agree in most respects with previous phase-field studies. ${ }^{43,44}$ However, our unified framework for studying reversible and irreversible aggregation by varying both the capillary length and the equilibrium density of adatoms provides an important extension of these studies. ${ }^{45}$ The main advantage of the phase-field method over level-set simulations is that no change in algorithm is needed to simulate these cases. Furthermore, compared with KMC simulations, there is no appreciable increase in the computational overhead for simulations of reversible growth, an advantage shared with the level-set method. ${ }^{37}$

The model we have used is the most basic for describing epitaxial growth: a uniform particle source which deposits single monatomic species onto a heated surface, which then diffuse isotropically until the nucleation of a new island or capture by an existing island. This model has been used to great effect for understanding a wide range of epitaxial phenomena, but there are areas where it can be improved.

For irreversible aggregation, the island-size distribution indicates that there are too many small islands, which is due to our nucleation algorithm, and is also seen in level-set calculations. ${ }^{72}$ This does not affect the scaling of island density with $R$, but may delay the onset of the saturation regime. If we replaced our uniform deposition flux with random deposition of atoms, ${ }^{43-45}$ then a detailed calibration against KMC simulations would enable the direct calculation of binding energies of critical islands, which would provide a way of determining detachment barriers. Some progress along these lines was reported in Ref. 45.

There are also many scenarios that require additional processes and interactions to be considered, such as stepped surfaces,${ }^{47-49}$ step-edge barriers, ${ }^{43}$ and the mobility of dimers, small clusters, or islands. ${ }^{52,89-93}$ All of these studies benefit from a large body of KMC simulations and, in some cases, analyses based on rate equations to identify appropriate scaling laws. The mobility of dimers has yet to be incorporated into the phase-field description, but is readily done, for example, with a diffusion constant $D(A)=D_{0} A^{-\mu}$ for islands of size $\mu>0$.
Another extension is deposition and decomposition polyatomic molecules that contain the atomic constituents of the growing film, which is the basis of metal-organic vaporphase epitaxy. The application of the phase field method to this growth scenario on surfaces patterned with V-grooves or inverted pyramids, ${ }^{94}$ whose facets have different properties, would enable computations on the scale of nanostructure arrays that would complement modeling based on reaction-diffusion equations. ${ }^{95}$

\section{ACKNOWLEDGMENTS}

J.P.d.B. was supported through a studentship in the Centre for Doctoral Training on the Theory and Simulation of Materials at Imperial College London funded by the Engineering and Physical Sciences Research Council (EPSRC) under Grant No. EP/G036888/. J.P.d.B. thanks F. Ming for helpful discussions, and D.D.V. thanks Andy Zangwill for a useful correspondence and for a critical reading of an earlier version of this manuscript.

${ }^{1}$ L. Royer, Bull. Soc. Fr. Miner. Crystallog. 51, 7 (1928).

${ }^{2}$ V. A. Shchukin, N. N. Ledentsov, and D. Bimberg, Epitaxy of Nanostructures (Springer, Berlin, 2003).

${ }^{3}$ Low-Dimensional Semiconductor Structures: Fundamentals and Device Applications, K. W. J. Barnham and D. D. Vvedensky (Cambridge University Press, Cambridge, UK, 2001).

${ }^{4}$ J. Michel, J. Liu, and L. C. Kimerling, Nat. Photonics 4, 527 (2010).

${ }^{5}$ K. S. Novoselov, A. K. Geim, S. V. Morozov, D. Jiang, Y. Zhang, S. V. Dubonos, I. V. Grigorieva, and A. A. Firsov, Science 306, 666 (2004).

${ }^{6}$ C. Berger, Z. Song, T. Li, X. Li, A. Y. Ogbazghi, R. Feng, Z. Dai, A. N. Marchenkov, E. H. Conrad, P. N. First, and W. A. J. de Heer, Phys. Chem. B 108, 19912 (2004).

${ }^{7}$ H. Tetlow, J. P. de Boer, I. J. Ford, D. D. Vvedensky, J. Coraux, and L. Kantorovich, Phys. Rep. 542, 195 (2014).

${ }^{8}$ A.-L. Barabási and H. E. Stanley, Fractal Concepts in Surface Growth (Cambridge University Press, Cambridge, UK, 1995).

${ }^{9}$ A. Pimpinelli and J. Villain, Physics of Crystal Growth (Cambridge University Press, Cambridge, UK, 1998).

${ }^{10} \mathrm{~J}$. A. Venables, Introduction to Surface and Thin Film Processes (Cambridge University Press, Cambridge, UK, 2000).

${ }^{11}$ T. Michely and J. Krug, Islands, Mounds and Atoms: Patterns and Processes in Crystal Growth Far from Equilibrium (Springer, Berlin, 2003).

${ }^{12}$ J. E. Northrup and S. Froyen, Phys. Rev. B 50, 2015(R) (1994).

${ }^{13}$ C. G. Morgan, P. Kratzer, and M. Scheffler Phys, Rev. Lett. 82, 4886 (1999).

${ }^{14}$ G. Henkelman, B. P. Uberuaga, and H. Jónsson, J. Chem. Phys. 113, 9901 (2000).

${ }^{15}$ Q.-M. Zhang, C. Roland, P. Bogusławski, and J. Bernholc, Phys. Rev. Lett. 75, 101 (1995).

${ }^{16}$ K. Hara, M. Ikeda, K. Terakura, M. Mikami, Y. Tago, and T. Oguchi, Phys. Rev. B 39, 9476 (1989).

${ }^{17}$ A. Madhukar and S. V. Ghaisas, Crit. Rev. Solid State Mater. Sci. 14, 1 (1988).

${ }^{18}$ K. A. Fichthorn and W. H. Weinberg, J. Chem. Phys. 95, 1090 (1991).

${ }^{19}$ P. Šmilauer and D. D. Vvedensky, Phys. Rev. B 48, 17603 (1993).

${ }^{20}$ H. Metiu, Y.-T. Lu, and Z. Zhang, Science 255, 1088 (1992).

${ }^{21}$ D. D. Vvedensky, J. Phys.: Condens. Matter 16, R1537 (2004).

${ }^{22}$ B. A. Joyce, Rep. Prog. Phys. 48, 1637 (1985).

${ }^{23}$ E. Loginova, N. C. Bartelt, P. J. Feibelman, and K. F. McCarty, New J. Phys. 10, 093026 (2008).

${ }^{24}$ E. Loginova, N. C. Bartelt, P. J. Feibelman, and K. F. McCarty, New J. Phys. 11, 063046 (2009).

${ }^{25}$ V. I. Tokar and H. Dreyssé, Phys. Rev. E 77, 066705 (2008).

${ }^{26}$ V. I. Tokar and H. Dreyssé, Phys. Rev. B 80, 161403(R) (2009).

${ }^{27}$ J. A. Venables, G. D. T. Spiller, and M. Hanbücken, Rep. Prog. Phys. 47, 399 (1984).

${ }^{28}$ G. S. Bales and D. C. Chrzan, Phys. Rev. B 50, 6057 (1994). 
${ }^{29}$ A. Zangwill and D. D. Vvedensky, Nano Lett. 11, 2092 (2011).

${ }^{30}$ T. P. Schulze, J. Crystal Growth 263, 605 (2004).

${ }^{31}$ G. Russo, L. M. Sander, and P. Smereka, Phys. Rev. B 69, 121406(R) (2004).

${ }^{32}$ J. P. DeVita and L. M. Sander. and P. Smereka, Phys. Rev. B 72, 205421 (2005).

${ }^{33}$ R. E. Caflisch, M. F. Gyure, B. Merriman, S. J. Osher, C. Ratsch, D. D. Vvedensky, and J. J. Zinck, Appl. Math. Lett. 12, 13 (1999).

${ }^{34}$ W. K. Burton, N. Cabrera, and F. C. Frank, Philos. Trans. R. Soc., A 243, 299 (1951)

${ }^{35}$ J. A. Sethian, Level Set Methods and Fast Marching Methods, 2nd ed. (Cambridge University Press, Cambridge, UK, 1999).

${ }^{36}$ S. Osher and R. Fedkiw, Level Set Methods and Dynamic Implicit Surfaces, Volume 153 of Applied Mathematical Sciences (Springer, New York, 2003).

${ }^{37}$ M. Petersen, C. Ratsch, R. E. Caflisch, and A. Zangwill, Phys. Rev. E 64, 061602 (2001).

${ }^{38}$ M. Petersen, A. Zangwill, and C. Ratsch, Surf. Sci. 536, 55 (2003).

${ }^{39}$ C. Ratsch, J. DeVita, and P. Smereka, Phys. Rev. B 80, 155309 (2009).

${ }^{40}$ J. S. Langer, in Directions in Condensed Matter Physics, edited by

G. Grinstein and G. Mazenko (World Scientific, Philadelphia, PA, 1986), pp. 164186.

${ }^{41}$ H. Emmerich, Adv. Phys. 57, 1 (2008)

${ }^{42}$ I. Singer-Loginova and H. M. Singer, Rep. Prog. Phys. 71, 106501 (2008).

${ }^{43}$ Y.-M. Yu and B.-G. Liu, Phys. Rev. E 69, 021601 (2004).

${ }^{44}$ F. Ming and A. Zangwill, Phys. Rev. B 81, 235431 (2010).

${ }^{45}$ X. L. Dong, H. Xing, C. L. Chen, J. Y. Wang, and K. X. Jin, Phys. Lett. A 379, 2452 (2015).

${ }^{46}$ Y.-M. Yu and B.-G. Liu, Phys. Rev. B 77, 195327 (2008).

${ }^{47}$ Y.-M. Yu and B.-G. Liu, Phys. Rev. B 70, 205414 (2004).

${ }^{48}$ Y.-M. Yu and B.-G. Liu, Phys. Rev. B 73, 035416 (2006).

${ }^{49}$ Z. Hu, J. S. Lowengrub, S. M. Wise, and A. Voigt, Physics D 241, 77 (2012).

${ }^{50}$ Y.-M. Yu, B.-G. Liu, and A. Voigt, Phys. Rev. B 79, 235317 (2009).

${ }^{51}$ M. Albani, R. Bergamaschini, and F. Montalenti, Phys. Rev. B 94, 075303 (2016).

${ }^{52}$ J. W. Evans, P. A. Thiel, and M. C. Bartelt, Surf. Sci. Rep. 61, 1 (2006).

${ }^{53}$ J. A. Venables, Philos. Mag. 27, 697 (1973).

${ }^{54}$ T. Vicsek and F. Family, Phys. Rev. Lett. 52, 1669 (1984).

${ }^{55}$ M. C. Bartelt and J. W. Evans, Phys. Rev. B 54, R17359 (1996).

${ }^{56}$ D. D. Vvedensky, Phys. Rev. B 62, 15435 (2000).

${ }^{57}$ M. C. Bartelt and J. W. Evans, Phys. Rev. B 46, 12675 (1992).

${ }^{58}$ S. A. Barnett and A. Rocket, Surf. Sci. 198, 133 (1988).

${ }^{59}$ T. Shitara, D. D. Vvedensky, M. R. Wilby, J. Zhang, J. H. Neave, and B. A. Joyce, Phys. Rev. B 46, 6815 (1992).

${ }^{60}$ T. Volkmann, M. Ahr, and M. Biehl, Phys. Rev. B 69, 165303 (2004).

${ }^{61}$ C. Ratsch and J. A. Venables, J. Vac. Sci. Technol., A 21, S96 (2003).

${ }^{62}$ J. A. Stroscio and D. T. Pierce, Phys. Rev. B 49, 8522 (1994).

${ }^{63}$ A. R. Avery, H. T. Dobbs, D. M. Holmes, B. A. Joyce, and D. D. Vvedensky, Phys. Rev. Lett. 79, 3938 (1997).

${ }^{64}$ F. J. Meyer zu Heringdorf, M. C. Reuter, and R. M. Tromp, Nature 412, 517 (2001).
${ }^{65}$ L. D. Landau and E. M. Lifshitz, Statistical Physics (Addison-Wesley, Reading, MA, 1969), Chap. 15.

${ }^{66}$ N. C. Bartelt, W. Theis, and R. M. Tromp, Phys. Rev. B 54, 11741 (1996).

${ }^{67}$ F. Liu and H. Metiu, Phys. Rev. E 49, 2601 (1994).

${ }^{68}$ C. A. Haselwandter and D. D. Vvedensky, Europhys. Lett. 77, 38004 (2007).

${ }^{69}$ L. Raymond, A. Verga, and D. D. Vvedensky, Mater. Sci. Semicond. Process. 12, 2 (2009).

${ }^{70}$ A. Karma and W.-J. Rappel, Phys. Rev. E 53, R3017 (1996).

${ }^{71}$ Our expression differs from that in Ref. 28 in two respects. The argument of the logarithm is manifestly dimensionless, and our numerical factor is $\pi$, rather than $\sqrt{\pi}$. Both differences can be explained by using the smallargument limit for $s=1$ in (16) of Ref. 28 to obtain $\sigma_{1}=-4 \pi / \ln \left(R_{1}^{2} \xi^{-2}\right)$, where $R_{1}$ is the radius corresponding to the area of an adatom and $\xi$ is the adatom extinction length, given by (13) of Ref. 28. Using $\pi R_{1}^{2}=\Omega$ and (10) of Ref. 28 to solve for $\xi^{-2}$ yields our expression for $\sigma$ in (18) in the main text.

${ }^{72}$ C. Ratsch, M. F. Gyure, S. Chen, M. Kang, and D. D. Vvedensky, Phys. Rev. B 61, R10598 (2000).

${ }^{73}$ A. Abramowitz and I. A. Stegun, Handbook of Mathematical Functions (Dover, New York, 1964), p. 1004.

${ }^{74}$ S. M. Cox and P. C. Matthews, J. Comput. Phys. 176, 430 (2002).

${ }^{75}$ J. A. Stroscio, D. T. Pierce, and R. A. Dragoset, Phys. Rev. Lett. 70, 3615 (1993).

${ }^{76}$ P. Stoltze, J. Phys.: Condens. Matter 6, 9495 (1994).

${ }^{77}$ M. C. Bartelt, L. S. Perkins, and J. W. Evans, Surf. Sci. 344, L1193 (1995).

${ }^{78}$ C. Ratsch, A. Zangwill, P. Śmilauer, and D. D. Vvedensky, Phys. Rev. Lett. 72, 3194 (1994).

${ }^{79}$ A. Karma and W.-J. Rappel, Phys. Rev. Lett. 77, 4050 (1996).

${ }^{80}$ A. Karma and W.-J. Rappel, Phys. Rev. E 57, 4323 (1998).

${ }^{81}$ C. Ratsch, P. Šmilauer, A. Zangwill, and D. D. Vvedensky, Surf. Sci. 329, L599 (1995).

${ }^{82}$ D. D. Chambliss and K. E. Johnson, Phys. Rev. B 50, 5012 (1994).

${ }^{83}$ J. G. Amar and F. Family, Phys. Rev. Lett. 74, 2066 (1995).

${ }^{84}$ A. Zangwill and E. Kaxiras, Surf. Sci. 326, L483 (1995).

${ }^{85}$ V. Cherepanov, S. Filimonov, J. Mysliveček, and B. Voigtländer, Phys. Rev. B 70, 085401 (2004).

${ }^{86}$ F. G. Gibou, C. Ratsch, M. F. Gyure, S. Chen, and R. E. Caflisch, Phys. Rev. 63, 115401 (2001).

${ }^{87}$ J. G. Amar, F. Family, and P. M. Lam, Phys. Rev. B 50, 8781 (1994).

${ }^{88}$ T. J. Oliveira and F. D. A. Aarao Reis, Phys. Rev. B 87, 235430 (2013).

${ }^{89}$ J. Villain, A. Pimpinelli, L.-H. Tang, and D. Wolf, J. Phys. I 2, 2107 (1992).

${ }^{90}$ S. Liu, L. Bonig, and H. Metiu, Phys. Rev. B 52, 2907 (1995).

${ }^{91}$ M. C. Bartelt, S. Günther, E. Kopatzki, R. J. Behm, and J. W. Evans, Phys. Rev. B 53, 4099 (1996).

${ }^{92}$ I. Furman and O. Biham, Phys. Rev. B 55, 7917 (1997).

${ }^{93}$ P. A. Mulheran and D. A. Robbie, Phys. Rev. B 64, 115402 (2001).

${ }^{94}$ E. Pelucchi, S. T. Moroni, V. Dimastrodonato, and D. D. Vvedensky, J. Mater. Sci.: Mater. Electron. 29, 952 (2018).

${ }^{95}$ V. Dimastrodonato, E. Pelucchi, and D. D. Vvedensky, Phys. Rev. Lett. 108, 256102 (2012). 